\title{
Spectral Analysis of the LMC [WC] Star SMP 61
}

\author{
G. Gräfener ${ }^{1}$, W.-R. Hamann ${ }^{1} \&$ M. Peña ${ }^{2}$ \\ ${ }^{1}$ Department of Physics, University of Potsdam, Germany \\ ${ }^{2} U N A M$, México
}

\begin{abstract}
HST UV and optical spectra of the early-type [WC] star SMP 61 in the LMC are analyzed by means of line blanketed non-LTE models for expanding atmospheres. The known distance to the LMC allows a reliable determination of the stellar parameters. The low iron surface abundance of the object possibly indicates a preceding evolution through a very late thermal pulse (VLTP).
\end{abstract}

HST spectroscopy of LMC stars allows for the determination of reliable stellar parameters (especially $L_{\star}, R_{\star}$ and $\dot{M}$ ) due to the known distance and the moderate interstellar extinction towards the LMC. A disadvantage is the large distance, which leads to a strong nebular contamination because the nebula of SMP 61 is so compact that it is nearly covered by the spectroscopic slit. For the subtraction of the nebular contribution we use a theoretical nebular continuum from Peña et al. (1997) to compensate the Balmer jump, which is clearly visible in the observation.

The spectral analysis of SMP 61 is performed with up-to-date non-LTE models for expanding atmospheres which account for iron group line blanketing and clumping (see e.g. Hamann et al., these proceedings; Gräfener et al. 2002). Complex model atoms of $\mathrm{He}, \mathrm{C}, \mathrm{O}, \mathrm{Si}$ and the iron group are considered. The spectral fit is presented in Hamann et al. (these proceedings). The derived luminosity $\left(L_{\star}=10^{3.9} \mathrm{~L}_{\odot}\right.$ with $\left.E_{B-V}=0.122 \mathrm{mag}\right)$ corresponds exactly to the standard value, which is expected for a central star with $M_{\star} \approx 0.6 \mathrm{M}_{\odot}$. With a clumping factor of $D=4$ (corresponding to a volume filling factor of $1 / 4$ ) we obtain a mass loss rate of $\dot{M}=10^{-6.17} \mathrm{M}_{\odot} \mathrm{yr}^{-1}$ while the terminal wind velocity is $v_{\infty}=1400 \mathrm{~km} / \mathrm{s}$.

Apart from a narrower line width, the spectral appearance of SMP 61 is very similar to the well known massive WC 5 star WR 111 (Hillier \& Miller 1999, Gräfener et al. 2002). Consequently, we derive a similar stellar temperature and surface composition $\left(T_{\star}=87.5 \mathrm{kK}\right.$ with $R_{\star}=0.39 R_{\odot}$ and $X_{\mathrm{C}}=0.5, X_{\mathrm{O}}=$ $0.03)$. The carbon mass fraction is in accordance with recent results of Crowther et al. (these proceedings) who derives similar values for a representative sample of galactic [WC] central stars.

As demonstrated in Fig. 1, our model with standard iron abundance (1/4 solar for LMC central stars) produces too strong iron features in the UV (the so-called "iron forest" around $1300 \AA)$. A model with lower iron abundance $(1 / 30$ solar) shows much weaker iron features which are below the detection limit of the observation. A detailed analysis yields an upper limit for the iron abundance of 


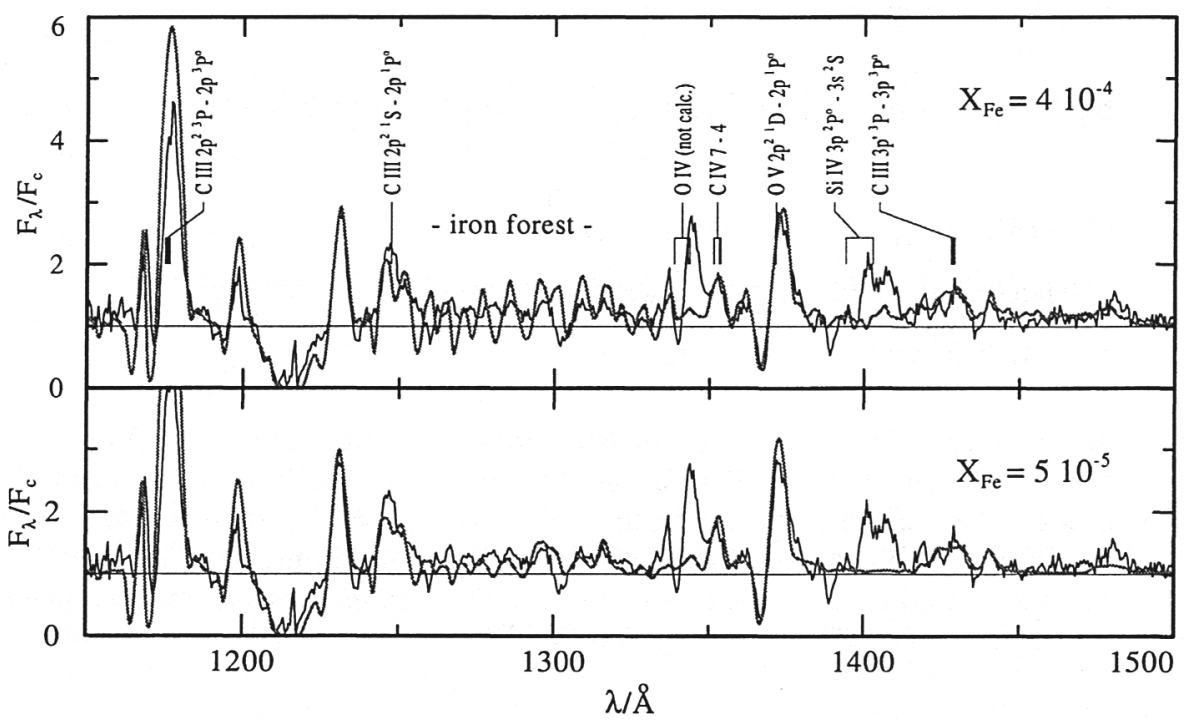

Figure 1. The iron forest of the HST UV spectrum (thin line) in comparison to model calculations (grey) with $X_{\mathrm{Fe}}=410^{-4}(1 / 4$ solar) and $X_{\mathrm{Fe}}=510^{-5}(1 / 30$ solar $)$. The model with the "standard" value of $X_{\mathrm{Fe}}=410^{-4}$ clearly shows iron features that are not observed.

$\approx 1 / 15$ solar. This result is in line with the detection of a strong iron depletion for A 78, a PG 1159-type central star and probable descendant of a [WC] central star (Werner et al., these proceedings). As pointed out by Herwig, Lugaro \& Werner (these proceedings) such an iron deficiency can be explained by the destruction of $\mathrm{Fe}$ in the neutron-capture nucleosynthesis during a VLTP.

\section{References}

Gräfener G., Koesterke L., Hamann W.-R. 2002, submitted to A\&A

Hillier D. J., \& Miller D. L. 1999, ApJ 519, 354

Peña M., Ruiz M. T., Torres-Peimbert S. 1997, A\&A 324, 674 